\title{
To Assess and Correlate Cardiorespiratory Adaptations in Endurance-Trained Cyclist and Recreational Cyclist
}

\section{Ananya Mandal *1, Gaurav Kadyan ${ }^{2}$.}

${ }^{* 1}$ Physiotherapist (Masters in Sports Physiotherapy, student), Indian Spinal Injuries Centre-Institute of Rehabilitation Sciences, Sector C, Vasant Kunj, New Delhi, Delhi 110070, India.

${ }^{2}$ Assistant Professor, Indian Spinal Injuries Centre-Institute of Rehabilitation Sciences, Sector C, Vasant Kunj, New Delhi, Delhi 110070, India.

\section{ABSTRACT}

Background: This cross-sectional study aimed to compare and correlate HRV indices, body composition, and aerobic power (VO2max) in recreational cyclists and trained endurance (road) cyclists. 47 male cyclists participated in the study (age: $20.32 \pm 0.365$; height: $171.50 \pm 1.117$; BMI: $23.00 \pm 0.47$ ) divided into 3 groups (Group 1: Recreational cyclist; group 2: Delhi Cyclist, playing at State/National level; and group 3: IGI cyclist, playing at National/International level).

Methods: The participants underwent body composition analysis (BMI, Subcutaneous whole body, body density, body fat \%, lean body mass) and anthropometry profiling. HRV was recorded (RMSSD, pNN50\%, SDNN, $\mathrm{LH} / \mathrm{HF}$ ratio) at resting state ( 5 minutes) using the Heartware Shimmer ECG device. Later 3 minutes McArdle step test was done to measure VO2 max.

Result: The VO2max $\left(F_{2,44}=22.821, P<0.001\right)$ and Body fat \% (7-Fold) $\left(F_{2,44}=6.772, P=0.003\right)$ differed significantly between the 3 groups of cyclists. A highly significant negative correlation was seen between cycling experience in years with Resting HR and LF/HF ratio $(p<0.01)$ with their $r$ - values at -.382 and -.448 respectively, and statistically significant negative correlation was indicated with Body fat\% (7-fold), $r=-.322(p<0.05)$.

Conclusion: Significant differences were seen in the aerobic capacity (VO2max) between all the 3 groups of cyclists. Improved VO2max in trained road cyclists can be particularly due to the nature of cycling (endurance) training, overall training volume, and reduction in the body fat $\%$ and fat mass, which further enhances the adaptations to autonomic control.

KEY WORDS: adaptation, autonomic nervous system, body composition, cardiorespiratory, cyclist, endurance, HRV, recreational, road cycling, VO2max.

Address for correspondence: Ananya Mandal, Physiotherapist (Masters in Sports Physiotherapy, student), Indian Spinal Injuries Centre-Institute of Rehabilitation Sciences, Sector C, Vasant Kunj, New Delhi, Delhi 110070, India. Contact no : (M) +91 9910566320

E-Mail: ananyamandal6@gmail.com
Access this Article online
Journal Information

Quick Response code

International Journal of Physiotherapy and Research ISSN (E) 2321-1822 | ISSN (P) 2321-8975 https://www.ijmhr.org/ijpr.html

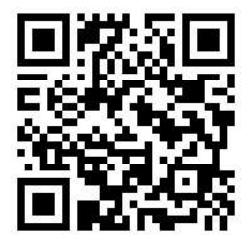

DOI: $10.16965 /$ ijpr.2021.193
DOI-Prefix: https://dx.doi.org/10.16965/ijpr

\section{Article Information}

Received: 29 Aug 2021

Peer Review: 30 Aug 2021

Revised: 07 Oct 2021
Accepted: 26 Oct 2021

Published (O): 11 Dec 2021

Published (P): 11 Dec 2021

\section{INTRODUCTION}

Road cycling is a high endurance sport, with an elite cyclist riding approximately 30000 to 35000 kilometers each year during training and competition. [1,2] The performance in cycling is mainly determined by maximal aerobic power over long-lasting distances and anaerobic power to sprint fast during the close gaps to finish the race first. [3] Professional cycling is characterized by two possible competition formats, i.e., time-trial (individual or small teams) and mass-start racing, which is found 
in most cycling disciplines (road, track, BMX, cyclo-cross, and mountain biking) $[2,4]$. Riders competing in the mass start, multi-stage races are characterized by stochastic shifts in work rate and speed. They are primarily dependent on the varying terrain (i.e., level, uphill and downhill roads) and competitive situations (i.e., individually or drafting behind other cyclists). In individual and team time trials, a rider or team sustains the highest average power output possible for the duration of a race $[1,5]$. The cyclist's power output is used during competitive road events to overcome the aerodynamic and rolling resistance [5]. Air resistance while riding on flat roads and gravity while climbing uphill or an elevated road thus acts as the main resistive forces that influence the energy cost of road cycling. This energy expenditure to overcome resistive forces when climbing uphill or elevated road increases with an increase in body weight of the cyclist [6]. Male professional road cycling competition is composed of 1-day, 4- to 10-day, and 3-week races. The events are lasting between 1 hour (e.g., the time trial in the World Championships) and 100 hours (e.g., the Tour de France) [2].

Professional cyclists must be able to tolerate high workloads for extended periods ( 3 weeks) during major tour races such as the Giro d'Italia, Tour de France, and VueltaaEspa na [7].

Heart rate variability (HRV) is a daily routine non-invasive method used to monitor the status of the autonomic nervous control and understand the physiological adaptations of the body in amateur and trained athletes [8]. This tool helps track and record the training status, "exercise readiness," and post-exercise fatigue in individual athletes. Having relevant indicators of the exercise stress-recovery status could be a real advantage for individual athlete's monitoring to avoid the state of overreaching and overtraining $[8,9]$-the adaptive capacity increases as the HRV increases, where low stress acts as a desirable condition. The HRV tends to decrease with age when there are pathological processes, in the presence of drug use, precompetitive stress, over-training, and before competitive matches
[10]. Heart-rate recovery is mainly attributed to vagal reaction immediately after the exercise. So, there may be a probability that an adaptation of the cardiac autonomic nervous system (ANS) to endurance training contributes to a more rapid heart-rate recovery after exercise $[11,12]$

Heart rate variability (HRV) has been proposed as an effective tool for prescribing training in running, cross-country skiing, and road cycling. The study by Jayaloyes et al. showed more significant increments in performance in well-trained cyclists on an HRV-guided training program compared with a standardized program without HRV [13].

VO2max reflects the physical fitness of an individual having the athletic capacity [14]. Male professional road cyclists have very high aerobic capacities, both at maximal and submaximal exercise intensities. With the decrease in body fat, there is an increase in aerobic fitness $[15,16]$. Mountain biking competition involves more variable power demands than road cycling, with activity frequently performed at high and maximal heart rate levels, oxygen uptake, and blood lactate concentrations [17]. Prolonged, intense endurance training, when conducted over many seasons, may induce neuromuscular adaptations that enable elite riders to recruit a more significant number of muscle fibres and spread the power production over a large active muscle mass while pedaling less well-trained cyclists [1]. Combining resistance and aerobic training repeatedly improved measures of strength and endurance in untrained individuals; this may be possible because of increased overall fitness. But for well-trained endurance road cyclists, it is less specific if improvements in strength from resistance training result in improved performance such as time trials of varying distances, time to exhaustion, or 1-hour cycle tests [18]. The anthropometric characteristics of a professional cyclist differ from other professional athletes due to their maximal use of the lower limbs. During cycling, the quadriceps and biceps femoris muscles are responsible factors for high performance. Sprint cyclist has a greater thigh girth and more 
muscle mass than endurance road cyclist [5].

Various studies of sprint cyclists and studies related to triathletes focus on correlation and comparing body composition, aerobic capacity, and heart rate variability.

So, the present study aimed to evaluate the relationships between body composition, aerobic capacity, and heart rate variability between endurance-trained and recreational road cyclists.

\section{METHODS}

Participants: 47 male cyclists participated in the study (age: $20.32 \pm 0.365$; height: $171.50 \pm$ 1.117; BMI: $23.00 \pm 0.47$ ) divided into 3 groups (group 1: Recreational cyclist, $n=15$; group 2: Delhi Cyclist, playing at State/National level, $\mathrm{n}=17$; and group 3: IGI cyclist, playing at National/International level, $\mathrm{n}=15$ ). Group 2 and 3 were trained endurance cyclists, while group 1 recreational cyclists were untrained and had no previous experience in cycling competitions.

Before obtaining informed consent, all the participants were acquainted with the research procedures, requirements, benefits, and risks. Written informed consent was taken from all of the subjects. This study was approved by Institutional Ethics Committee (IEC) of Indian Spinal Injuries Centre, Vasant Kunj, New Delhi. (ISIC/RP/2021/24).

The participants did not present with any acute or chronic disease; the trained endurance cyclists group systematically trained and prepared for the 2021 National Cycling Championship in Mumbai. Trained participants were told not to exercise intensely or engage in structured endurance or strength training on the day before assessment.

The cyclists were called for the testing on their recovery day. The time of assessment was scheduled in the morning hours between 9 AM -12 PM. There was a single evaluation done for each cyclist, and it was carried out in a quiet and comfortable room (Sports Lab) of ISIC-IRS and IGI stadium (SAI), with temperature maintained around 25 to $28^{\circ} \mathrm{C}$. Blood pressure was measured with Omron Sphygmomanometer. Other general details were filled in, and the subjects completed the Stress
Symptom Checklist [19] to screen their stress levels and only then was heart rate variability (HRV) measured under controlled circumstances. Subjects were pre-informed for not eating or drinking caffeinated beverages at least two hours before the testing to prevent alteration in heart rate. After the HRV test, physical fitness variables were also measured, including body mass, height, and body mass index (BMI), skinfolds for defining the percentage of body fat (BF), and later aerobic capacity (VO2max) was estimated with an indirect field test: 3 min. McArdle step test [20].

Measures of HRV: The HRV measurements were performed using a Heart wear Shimmer ECG device by EsMedTek, Consensys software for recording, and SinusCor for HRV analysis. The approach followed the recommendations of the Task Force [21].

The HRV was recorded in a sitting position. All the subjects were instructed to maintain a calm and normal breathing pattern and temperature of the room. To acquire a good quality ECG signal and to minimize the likelihood of signal interference from electrodes falling off or sporadic contact caused by hairs or dirt particles, it was ensured that all the participants shaved off their chest hair for an excellent electrode to skin contact. Also, the skin was cleaned with sanitizer to remove oils and sweat to avoid interference with the signal. The 5 electrodes were taken in which the two electrodes were placed at $2 \mathrm{~cm}$ below the clavicle (mid-clavicular line) on the right and left side. Rest two electrodes were placed two-finger breadth above the ASIS on the right and left sides. The remaining one electrode was placed over the apex beat, usually confirmed via stethoscope, although its reference point is $4-5^{\text {th }}$ intercostal space. The machine was connected with electrodes and connected to Consensys software via Bluetooth on the laptop, and HRV was recorded. The First 2 Minutes of ECG were recorded for observation, and then the actual ECG was recorded for 5 minutes. Following parameters of HRV were analyzed: Mean and standard deviation of heart rate (HR) in beats per minute (bpm), mean RR interval (RR), square root mean of successive differences 
between normal RR intervals (RMSSD), standard deviation of NN intervals (SDNN), low frequency spectral (LF) component, highfrequency spectral component (HF), low frequency/ high frequency (LF/HF) ratio.

Anthropometry profiling and body composition analysis: Every participant was asked to wear light clothing (shorts) and no shoes and socks during measurements. Anthropometry tape- Lufkin (W606PM) was used to measure the waist, hip, abdominal, thighs, and calves circumferences. The tape has an accuracy of $\pm 1 \mathrm{~mm}$. Body fat percentage was estimated using seven site skinfold thickness technique, with a scientific Skinfold Calliper- Harpenden calipers by ISAK. It has the accuracy to the nearest $0.1 \mathrm{~mm}$. The anatomical skinfold sites used were: the chest, abdominal, thigh, triceps, suprailiac, subscapular, and axilla. Estimation of body density followed the equation that has been validated for males aged between 18 to 61 years [3]

\section{7-site skinfold equation for}

Body Density $=1.112-(0.00043499 x$ sum of skinfolds $)+(0.00000055 \times$ square of the sum of skinfold sites) - (0.00028826 $x$ age)

Further, Body fat percentage was estimated using the Siri equation:

[\%Body fat $=$ (495 / body density) -450 ] and then Fat mass was calculated applying the equation:

fat mass $=($ body mass $\times \%$ body fat $) / 100]$. Lastly, Lean body mass was determined as body mass " fat mass).

The height was measured to the nearest 0.1 $\mathrm{cm}$ using an anthropometry rod, while body mass and body fat percentage was measured using Bioelectrical impedance analysis (Body Composition Monitor Model HBF-375 machine, Omron). Two readings were taken for each variable, and then the average values were used to perform the statistical analysis.

McArdle step test: All of the subjects completed a 3- minute McArdle step test on a Step of 16.25 inch $(41.3 \mathrm{~cm})$ height according to the procedures suggested by Kumar and Goswami, (2019). During the test, the subjects were verbally encouraged to give the maximum effort possible and not give up Int J Physiother Res 2021;9(6):4058-65. ISSN 2321-1822 before 3 minutes. The participants stepped up and down on the platform at a rate of 24 steps per minute using a four-step cadence, "up-up-down-down," following a metronome for 3 min.

On completing the test, the subject was made to stop the procedure immediately, and on recovery from 5 to $20 \mathrm{~s}$, the heartbeats had been counted.

This study comprised only male participants, so the estimation of VO2 max from the test results was calculated, using the formula given for men:

VO2 $\mathrm{max}(\mathrm{ml} / \mathrm{kg} / \mathrm{min})=111.33-(0.42 \times$ heart rate [bpm]). [20]

Statistical analysis: IBM SPSS (Statistical Package for the Social Sciences) software version 25 was used for statistical analysis. Spearman rank correlations were used to examine the correlation between resting heart rate, body composition, and HRV indices of all the subjects and each group of cyclists (Recreational cyclist, Delhi cyclist, and IGI cyclist) separately. Statistical comparisons among the different cyclist groups were accomplished using a one-way analysis of variance (ANOVA). In the event of a significant Levene statistic, the equal variance was not assumed. To check for individual differences between the groups (for pair-wise comparison), post-hoc comparison test using Dunnett's $\mathrm{T}_{3}$ was selected. A criterion alpha level of $p<0.05$ was used to determine the statistical significance.

\section{RESULTS}

Based on ANOVA results (Table 2), the VO2max $\left(F_{2,44}=22.821, P<0.001\right)$ and Body fat \% (7-Fold) $\left(F_{2,44}=6.772, P=0.003\right)$ of the groups differ significantly.

The mean scores of VO2max for Recreational cyclist ( $M=41.190, S D=6.755)$ was significantly different from IGI cyclists ( $M=54.966$, $\mathrm{SD}=5.776)$. Delhi cyclists $(\mathrm{M}=45.167, \mathrm{SD}=4.707)$ differed significantly from IGI cyclists. However, no significant differences were detected between Delhi cyclists and Recreational cyclists.

The mean scores of Body fat \% (7-Fold) for 
Recreational cyclist ( $M=15.775, \mathrm{SD}=3.685)$ was significantly different from IGI cyclists $(M=12.342, S D=1.215)$, while Delhi cyclists $(M=13.955, S D=2.189)$ differed significantly from IGI cyclist. However, no significant differences were seen between Delhi cyclists and Recreational cyclists.

The mean scores of Fat mass (7-Fold) $\left(F_{2,44}=3.270, P=0.47\right)$ for Recreational cyclists $(M=11.246, S D=4.467), I G \mid$ cyclists $(M=8.590$, $S D=1.261)$, and Delhi cyclists $(M=9.210$, $\mathrm{SD}=2.382$ ) showed no significant differences between the 3 groups of cyclists.
Table 1: Characteristics of the participants.

\begin{tabular}{lcc}
\hline & {$[X(S D)]$} & Min-Max \\
\hline AGE(yrs.) & $20.34(2.50)$ & $18-27$ \\
\hline BODY MASS(kg) & $67.2(10.41)$ & $46.6-96.0$ \\
\hline HEIGHT(cm) & $171.50(7.65)$ & $157.5-192.5$ \\
\hline BMI(kg/cm2) & $23.00(3.27)$ & $16.30-35.00$ \\
\hline BODY FAT (\%) & $14.02(2.85)$ & $9.61-21.25$ \\
FAT MASS(kg) & $9.66(3.13)$ & $4.94-18.44$ \\
\hline LEAN MASS(kg) & $58.06(7.95)$ & $40.53-77.88$ \\
VISCERAL FAT (\%) & $6.02(3.63)$ & $0.5-19.5$ \\
VO2max(ml/kg/min) & $47.025(8.043)$ & $26.07-63.87$ \\
HR rest. (bpm) & $72.70(11.30)$ & $53-98$ \\
CYCLING EXPERIENCE (yrs.) & $3.01(1.87)$ & $0.2-7.0$ \\
TRAINING DURATION (hrs/day) & $2.84(1.39)$ & $01-05$ \\
AVERAGE KILOMETERS (per day) & $65.32(33.28)$ & $5-100$ \\
\hline X M M
\end{tabular}

$\mathrm{X}=$ Mean SD= Standard deviation

Table 2: Results of descriptive statistics between the three groups.

\begin{tabular}{|c|c|c|c|c|c|c|c|c|}
\hline \multirow[t]{2}{*}{ GROUPS } & \multirow{3}{*}{$\rightarrow F(2,44)$} & \multirow[b]{3}{*}{$\mathbf{p}$} & \multirow{2}{*}{\multicolumn{2}{|c|}{$\begin{array}{l}\text { RECREATIONAL } \\
\text { CYCLIST }\left(\mathrm{N}_{1}=15\right)\end{array}$}} & \multirow{2}{*}{\multicolumn{2}{|c|}{$\begin{array}{c}\text { DELHI CYCLIST } \\
\left(\mathrm{N}_{2}=17\right)\end{array}$}} & \multirow{2}{*}{\multicolumn{2}{|c|}{$\begin{array}{c}\text { IGI CYCLIST } \\
\left(N_{3}=15\right)\end{array}$}} \\
\hline & & & & & & & & \\
\hline DEPENDENT VARIABLE & & & M & SD & M & SD & M & SD \\
\hline V02max & 22.821 & $<.001$ & 41.19 & 6.755 & 45.167 & 4.707 & 54.966 & 5.776 \\
\hline BODY FAT\%(7-Fold) & 6.772 & 0.003 & 15.775 & 3.685 & 13.955 & 2.189 & 12.342 & 1.215 \\
\hline FAT MASS(7-Fold) & 3.27 & 0.47 & 11.246 & 4.467 & 9.21 & 2.382 & 8.59 & 1.261 \\
\hline LEAN MASS(7-FOLD) & 1.478 & 0.239 & 57.68 & 10.405 & 56.019 & 7.659 & 60.769 & 4.404 \\
\hline LF/HF Ratio & 1.034 & 0.364 & 2.288 & 2.189 & 1.509 & 1.203 & 60.285 & 230.073 \\
\hline SDNN & 1.171 & 0.319 & 64.108 & 23.52 & 91.605 & 51.172 & 652.677 & 2125.77 \\
\hline RMSSD & 1.245 & 0.298 & 49.121 & 29.17 & 86.63 & 62.3 & 778.9 & 2550.4 \\
\hline
\end{tabular}

Table 3: Results shows the correlation analysis of Resting Heartrate, Body composition and HRV variables of the 3 group of cyclists (Recreational cyclist, Delhi cyclist and IGI cyclist) N=47

\begin{tabular}{|c|c|c|c|c|c|c|c|c|c|c|c|c|c|}
\hline$N=47$ & $\begin{array}{c}\text { RESTING } \\
\text { HEART } \\
\text { RATE }\end{array}$ & $\begin{array}{l}\text { CYCLING } \\
\text { EXPERIENC } \\
\text { E IN YEARS }\end{array}$ & $\begin{array}{l}\text { AVERAGE } \\
\text { KILOMETER } \\
\text { S PER DAY }\end{array}$ & $\begin{array}{c}\text { SUBCUTANE } \\
\text { OUS WHOLE } \\
\text { BODY FAT }\end{array}$ & $\begin{array}{c}\text { SKELETAL } \\
\text { WHOLE } \\
\text { BODY }\end{array}$ & $\begin{array}{c}\text { VISCERAL } \\
\text { FAT } \%\end{array}$ & $\begin{array}{l}\text { BODY FAT } \\
\% \text { (7-FOLD }\end{array}$ & $\begin{array}{l}\text { FAT MASS } \\
\text { (7-FOLD) }\end{array}$ & $\begin{array}{l}\text { LEAN } \\
\text { MASS(7- } \\
\text { FOLD }\end{array}$ & $\mathrm{VO2}_{\text {MAX }}$ & RMSSD & SDNN & $\begin{array}{l}\text { LF/HF } \\
\text { RATIO }\end{array}$ \\
\hline RESTING HEART RATE & & $-.382^{* *}$ & & & & & & & & & & & \\
\hline CYCLING EXPERIENCE IN YEARS & $-.382^{* *}$ & & & & & & $-322^{*}$ & & & & & & $-.448^{* *}$ \\
\hline AVERAGE KILOMETERS PER DAY & & $.567^{* *}$ & & & & & & & & $.607^{* *}$ & & $.306^{*}$ & \\
\hline SUBCUTANEOUS WHOLE BODY FAT & & & & & & $.732^{* *}$ & & & & & & & \\
\hline SKELETAL WHOLE BODY & & & & $-.937^{* *}$ & & $-.738^{* *}$ & $-.733^{* *}$ & $-.645^{* *}$ & & & & & \\
\hline VISCERAL FAT \% & & & & $.732^{* *}$ & $-.738^{* *}$ & & $.650^{* *}$ & $.690^{* *}$ & & & & & \\
\hline BODY FAT \%(7-FOLD) & & $-.322^{*}$ & $-.459^{* *}$ & & & & & & & $-.518^{* *}$ & & $-.304^{*}$ & $.302^{*}$ \\
\hline FAT MASS (7-FOLD) & & & $-.318^{*}$ & & & & $.907^{* *}$ & & & $-.384^{* *}$ & & & $.381^{* *}$ \\
\hline LEAN MASS(7-FOLD) & & & & & & & $.373^{*}$ & $.689^{* *}$ & & & & & $.306^{*}$ \\
\hline $\mathrm{VOZ}_{\text {MAX }}$ & & & $.607^{* *}$ & & & & $-.518^{* *}$ & $-.384^{* *}$ & & & & & \\
\hline RMSSD & $-.317^{*}$ & $.355^{*}$ & $.398^{* *}$ & & & & $-.343^{*}$ & $-.292^{*}$ & & & & $.899^{* *}$ & $-.579^{* *}$ \\
\hline SDNN & $-.335^{*}$ & & & & & & $-.304^{*}$ & & & & $.899^{* *}$ & & \\
\hline LF/HF RATIO & & $-.448^{* *}$ & & & & & $.302^{*}$ & $.381^{* *}$ & $.306^{*}$ & & $-.579^{* *}$ & & \\
\hline ** correlation is significant at the lev & of 0.01 leve & (2-tailed) & & & & & & & & & & & \\
\hline${ }^{*}$ Correlation is significant at the leve & f 0.05 level & (2-tailed) & & & & & & & & & & & \\
\hline
\end{tabular}

Also there is no significant difference of LEAN MASS (7-Fold) $\left(F_{2,44}=1.478, P=0.239\right), L F / H F$ ratio $\left(F_{2,44}=1.034, P=0.364\right)$ and SDNN values $\left(F_{2,44}=1.171, P=0.319\right)$ between the groups.

Based on the results (Table 3 ), correlation analysis revealed a highly significant negative correlation of $\mathrm{VO} 2 \mathrm{max}$ with Body fat\% and Fat mass with their respective $r$ - values at -0.518 and $-.384(p<0.01)$. While the highly significant positive correlation was seen with average riding kilometers per day $(p<0.01), r=.607$
A highly significant negative correlation was seen between cycling experience in years with Resting HR and LF/HF ratio $(p<0.01)$ with their $r$ - values at -.382 and -.448 respectively and statistically significant negative correlation was indicated with Body fat\% (7-fold), $r=-.322$ $(p<0.05)$.

Skeletal Whole-body showed a significantly strong negative correlation with Subcutaneous whole body fat, Visceral fat\%, Body fat \% ( 7-fold), and fat mass with their respective 
$r$ - values at $-.937,-.738,-.733$, and -.645 $(p<0.01)$. HRV Frequency domain: LF/HF ratio showed a strong significant negative correlation with RMSSD and cycling experience in years $(p<0.01)$ with their $r$ - values at -.579 and -.448 respectively, while a statistically significant positive correlation was seen with Body fat $\%$ and Lean mass $(p<0.05)$, their respective $r$-values at .302 and .306 respectively.

HRV Time domain: RMSSD showed a statistically significant negative correlation with Resting HR, Body fat\% and Fat mass $(p<0.05)$, with their respective $r$-values at $-.317,-.343$, and -.292 , respectively. While a highly significant positive correlation was seen with average riding kilometers per day $(p<0.05)$, $r=.398$

\section{DISCUSSION}

The study aimed to compare and correlate HRV indices, body composition, and aerobic power (VO2max) in recreational and trained endurance cyclists.

The study results showed significant differences in the aerobic capacity (VO2max) and Body fat\% between Recreational cyclists, Delhi Cyclists, and IGI cyclists. At the same time, there were no significant differences in Lean mass (7-fold), the LF/HF ratio (Frequency domain), and the SDNN (Time domain) between the 3 groups of cyclists.

The present study's findings showed significant differences in the aerobic capacity (VO2max) between all the 3 groups of cyclists. According to the study, the trained endurance cyclists showed better aerobic capacity than recreational cyclists. The finding can be explained by the overall factors like years of training, the kilometers of riding, and recovery days may greatly enhance cardiorespiratory fitness, which is highly demanding in road cycling, as the rider needs to sustain for long hours on the road without getting exhausted. In addition, on comparing aerobic capacity (VO2max) amongst the well-trained cyclists, this study also revealed that more improved aerobic capacity was seen in the IGI cyclist than the Delhi cyclist. This may be due to the effects of specific training interventions undertaken by the National/International level cyclists and their coaches. The result goes according to the other study by Lucia et al., which analyzed the VO2 kinetics in a group of top-level professional road cyclists, well-trained and amateur cyclists of a lower fitness level to determine the possible influence cardiorespiratory factors and lactate during a ramp protocol. The study concluded with the rise in $\mathrm{VO} 2$ in professional cyclists, and it was attenuated at the moderate to high workloads, contrary to the case in amateur riders. This may be possibly due to the adaptation of the professional cyclists to the higher demands of their training/ competition schedule [22]. So the previous study went in support of the present study.

In the present study, the riding kilometers showed a highly significant positive correlation with the aerobic capacity (VO2max) and RMSSD (one of the HRV parameter in Time domain), which signifies better aerobic capacity and autonomic adaptations in trained cyclists. While the results of the previous study by Oliveira et al. reported a negative correlation between aerobic capacity with the change in the RMSSD (i.e., ÄRMSSD) $(P=0.05)$ in trained cyclists, suggesting that physical fitness should be considered when evaluating autonomic control [10]. So the findings do not go with the other study.

The more years of endurance training of Delhi cyclists showed a negative correlation with the LF/HF ratio (an index of the autonomic modulation of the heart) and LFnu, signifying better cardiac adaptations in experienced cyclists. At the same time, the previous study by Oliveira et al. reported cycling competition as a stressful situation capable of altering autonomic parameters (considerable increase in the sympathetic drive) while anxiety was correlated with athletes' experience. The sympathetic modulation related to stress in trained athletes shows that the greater the athlete's training years and experience, the less anxiety they appear to encounter during stressful situations [10]. So the findings of the other study go in support of the present study.

The present study showed a statistically significant negative correlation of RMSSD with Resting HR, Body fat\% and Fat mass. In 
addition, the trained (endurance) cyclists had a higher RMSSD value than the recreational cyclist. A previous study by Arslan et al. has found no significant differences in the body fat $\%$, fat mass, and lean body mass between cyclists and triathletes. However, body fat percentage and fat mass were lower in triathletes than in cyclists. The study also revealed no significant differences in any heart rate variability responses between cyclists and triathletes. However, RMSSD (ms) was higher in cyclists compared to triathletes [3].

The result of the present study revealed a highly significant negative correlation of VO2max with Body fat\% and Fat mass. The study also showed that IGI cyclists, those playing at the National/International level, had higher VO2max values than Delhi cyclists (playing at State/National level) and Recreational cyclists. However, endurance-trained cyclists (Delhi cyclists and IGI cyclists) reported low levels of Body fat $\%$ and Fat mass compared to the Recreational cyclist. In addition, Body fat \% showed a strong significant positive correlation with visceral fat $\%$ with higher values seen in the Recreational cyclist. So the findings can be elaborated by the nature of cycling (endurance) training and overall training volume, which cause a reduction in the body fat $\%$ and fat mass in the trained cyclist, therefore sharing a strong relationship with aerobic capacity. These findings were similar to a previous study by Parikh et al., which reported an association of increased visceral fat with reduced aerobic fitness in Indian adolescents, while lower body fat and greater aerobic capacity are related to better autonomic control [16].

\section{CONCLUSION}

The present study establishes the correlation between aerobic capacity (VO2max), Body fat $\%$, fat mass, and riding kilometers per day. It is further established that some heart rate variability indices also correlate with body fat $\%$, lean mass, years of cycling experience (training). The results conclude significant differences in the aerobic capacity (VO2max) between all the 3 groups of cyclists. Improved VO2max in well-trained road cyclists can be mainly due to the nature of cycling (Endurance) training, overall training volume, and reduction in the body fat $\%$ and fat mass, which further enhances the adaptations to autonomic control. In conclusion, it could be inferred that cardiorespiratory fitness and body composition go hand in hand; to stay physically fit and mentally calm during road cycling events is very important for the cyclist to sustain long hours of pedalling.

\section{ACKNOWLEDGEMENTS}

We gratefully thank Dr. Shambhovi Mitra PhD Scholar (IIT Delhi), MPT (Cardiothoracic) Assistant Professor, ISIC Institute of Rehabilitation Sciences for her expert advice and constant guidance throughout the course of this work. We are also grateful to Dr. Prahlad Priyadarshi, Senior Sports Physiotherapist (SAI, IGI Stadium) for extending his unconditional support, guidance and assistance in the various phases of this study.

\section{Conflicts of interest: None}

\section{REFERENCES}

[1]. Hawley JA, Stepto NK. Adaptations to training in endurance cyclists: Implications for performance. Sport Med. 2001;31(7):511-20.

[2]. Mujika I, Padilla S. Physiological and performance characteristics of male professional road cyclists. Sport Med. 2001;31(7):479-87.

[3]. Arslan E, Aras D. Comparison of body composition, heart rate variability, aerobic and anaerobic performance between competitive cyclists and triathletes. J Phys Ther Sci 2(ref). 2016;28(4): 1325-9.

[4]. Phillips KE, Hopkins WG. Determinants of Cycling Performance: a Review of the Dimensions and Features Regulating Performance in Elite Cycling Competitions. Sport Med - Open. 2020;6(1).

[5]. van der Zwaard S, de Ruiter CJ, Jaspers RT, de Koning JJ. Anthropometric Clusters of Competitive Cyclists and Their Sprint and Endurance Performance. Front Physiol. 2019;10(October):1-10.

[6]. Mondal H, Mishra SP. Effect of BMI, body fat percentage and fat free mass on maximal oxygen consumption in healthy young adults. J Clin Diagnostic Res. 2017;11(6):CC17-20.

[7]. Earnest CP, Jurca R, Church TS, Chicharro JL, Hoyos J, Lucia A. Relation between physical exertion and heart rate variability characteristics in professional cyclists during the Tour of Spain. Br J Sports Med. 2004;38(5):568-75.

[8]. Dong JG. The role of heart rate variability in sports physiology (Review). Exp Ther Med. 2016;11(5):1531-6. 

ational Cyclist.

[9]. Barrero A, Le Cunuder A, Carrault G, Carré F, Schnell F, Le Douairon Lahaye S. Modeling Stress-Recovery Status Through Heart Rate Changes Along a Cycling Grand Tour. Front Neurosci. 2020;14(December):111.

[10]. Oliveira-Silva I, Silva VA, Cunha RM, Foster C. Autonomic changes induced by precompetitive stress in cyclists in relation to physical fitness and anxiety. PLoS ONE Discuss 1(ref). 2018;13(12):1-9.

[11]. Yamamoto K, Miyachi M, Saitoh T, Yoshioka A, Onodera S. Effects of endurance training on resting and post-exercise cardiac autonomic control. Med Sci Sports Exerc. 2001;33(9):1496-502.

[12]. Shaffer F, Ginsberg JP. An Overview of Heart Rate Variability Metrics and Norms. Front Public Heal. 2017;5(September):1-17.

[13]. Javaloyes A, Sarabia JM, Lamberts RP, Plews D, Moya-Ramon M. Training Prescription Guided by Heart Rate Variability Vs. Block Periodization in Well-Trained Cyclists. J strength Cond Res. 2020;34(6):1511-8.

[14]. Zhang R, Zhan L, Sun S, Peng W, Sun Y. Validity of a newly-designed rectilinear stepping ergometer submaximal exercise test to assess cardiorespiratory fitness. J Sport Sci Med. 2017;16(3):357-64.

[15]. Shete AN, Bute SS, Deshmukh PR. A study of VO2 max and body fat percentage in female athletes. J Clin Diagnostic Res. 2014;8(12):BC01-3.

[16]. Parikh S, Shah H, Singh S. Does visceral fat affect aerobic fitness in Indian adolescents of 18-19 years' age group? Natl J Physiol Pharm Pharmacol. 2017;(January 2017):1.

[17]. Hebisz P, Hebisz R, Borkowski J, Zaton M. Time of VO2max plateau and post-exercise oxygen consumption during incremental exercise testing in young mountain bike and road cyclists. Physiol Res. 2018;67(5):711-9.
[18]. Yamamoto LM, Klau JF, Casa DJ, Kraemer WJ, Armstrong LE, Maresh CM. The effects of resistance training on road cycling performance among highly trained cyclists: A systematic review. J Strength Cond Res. 2010;24(2):560-6.

[19]. Andreou E, Alexopoulos EC, Lionis C, Varvogli L, Gnardellis C, Chrousos GP, et al. Perceived Stress Scale: Reliability and validity study in Greece. Int J Environ Res Public Health. 2011;8(8):3287-98.

[20]. Kumar N, Goswami S. Comparison of Rockport one-mile walk test and McArdle step test for the prediction of VO 2 max. Saudi J Sport Med. $2019 ; 19(3): 82$.

[21]. Heart rate variability: standards of measurement, physiological interpretation and clinical use. Task Force of the European Society of Cardiology and the North American Society of Pacing and Electrophysiology. Circulation. 1996 Mar 1;93(5):1043-65.

[22]. Lucía A, Hoyos J, Santalla A, Pérez M, Chicharro JL. Kinetics of $\mathrm{VO} 2$ in professional cyclists. Med Sci Sports Exerc. 2002;34(2):320-5.

How to cite this article: Ananya Mandal, Gaurav Kadyan. To Assess and Correlate Cardiorespiratory Adaptations in Endurance-Trained Cyclist and Recreational Cyclist. Int J Physiother Res 2021;9(6):4058-4065. DOI: 10.16965/ijpr.2021.193 